\title{
深共熔溶剂热合成共轭配体修饰锆/钛氧团簇
}

金荣超

Department of Chemistry, Carnegie Mellon University, Pittsburgh, PA 15213, USA.

\section{Deep Eutectic Solvothermal Synthesis of Conjugate Ligands Functionalized Zr/Ti-Oxo Nanoclusters}

\author{
JIN Rongchao \\ Department of Chemistry, Carnegie Mellon University, Pittsburgh, PA 15213, USA. \\ Email: rongchao@andrew.cmu.edu
}

(a)

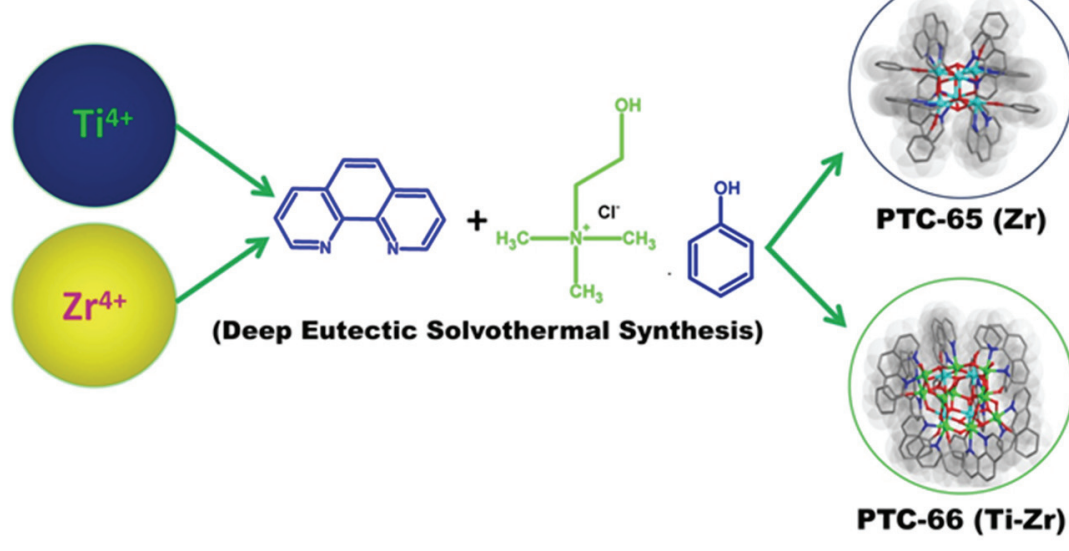

(b)

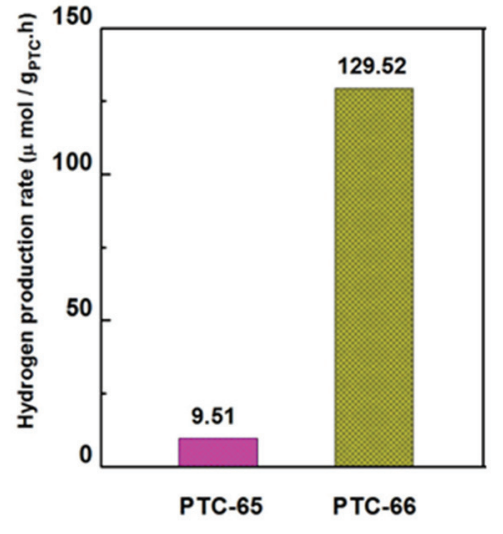

（a) 共轭配体修饰的锆/钛氧团簇的深共溶剂热合成方案; (b) 不同氧簇化合物的光催化分解水产氢活性对比。

注：为对比，共轭配体以蓝色结构式突出。为简化，晶体结构中，团簇上的氢原子已省去：

蓝色, $\mathrm{Zr}$ 原子; 绿色, $\mathrm{Ti}$ 原子; 红色, 氧原子; 蓝色, 氮原子; 灰色, 碳原子

金属氧簇化合物属于晶态分子材料, 可以通 过X射线单晶衍射分析获得精确原子结构, 以帮助 我们理解结构与性能之间的构效关系规律。特别 是钛氧团簇, 作为二氧化钛材料的重要模型化合 物, 近年来吸引了广泛的研究注意力 ${ }^{1-4}$ 。但是, 这 类高价金属离子极易水解, 而金属氧簇簇核的生 长又需要大量的氧桥, 这就使其合成具有较高难 度。目前常用的合成策略包括溶剂热合成、强酸水 溶液组装等 5,6 。另一方面, 为了调控这类氧簇化合 物的光吸收特性, 促进其在太阳能利用和清洁能 源方面的应用, 引入有机共轭配体将是有效方法,
而传统醇类溶剂对共轭配体较低的溶解能力则又 限制了这一方案的实施。针对这些问题, 中国科学 院福建物质结构研究所张磊、张健研究员等将深 共熔溶剂热合成引入到钛氧团簇领域 ${ }^{7}$, 近期又利 用这一合成方法成功制备了由邻菲罗啉、苯酚等 共轭配体修饰的 $\mathrm{Zr}_{6}$ 以及 $\mathrm{Ti}_{11} \mathrm{Zr}_{4}$ 团簇。他们通过巧 妙设计, 将苯酚与氯化胆碱按摩尔比 $2: 1$ 混合得到 一种离子液体, 其中苯酚既作为溶剂分子, 又可作 为簇核的共轭修饰配体。而这一新型离子液体对 邻菲罗啉等大共轭配体的溶解能力也会大大增 强, 有利于将其大量修饰到簇核表面。受益于此, 
他们所制备的 $\mathrm{Zr}_{6}$ 簇核由 6 个苯酚和 6 个邻菲罗啉配 体保护, 而 $\mathrm{Ti}_{11} \mathrm{Zr}_{4}$ 簇核则由 11 个苯酚和 8 个邻菲罗 啉配体保护。所以, 从某种意义上说, 这类团簇可 以看作是具有金属-氧无机核与共轭有机壳的一 类特殊分子核壳材料。

作为氧化物光催化材料的结构模型化合物, 这类金属氧簇化合物的分解水活性将具有重要研 究意义。但是, 通常制备的此类团簇表面都含有大 量的烷氧端基配体, 特别是醇做溶剂的情况下。而 这些烷氧端基配体很容易被水分子交换, 使得簇 核被进一步水解破坏, 极大限制了其光催化活性 测试。张磊、张健等人所引入的深共熔溶剂热合成 则可以很大程度上解决这个难题, 从他们所制备 的 $\mathrm{Zr}_{6}$ 以及 $\mathrm{Ti}_{11} \mathrm{Zr}_{4}$ 团簇的结构可以清晰地看到, 簇 核表面完全由共轭配体保护, 因此表现出较高的 水稳定性(水中浸泡 $24 \mathrm{~h}$ 保持团簇结构不变)。更加 有意思的是, 这两个团簇在光催化分解水测试的 过程中还表现出不同的分散特性, 其中 $\mathrm{Zr}_{6}$ 团簇保 持异相浑浊液状态, 而 $\mathrm{Ti}_{11} \mathrm{Zr}_{4}$ 团簇则形成高度分 散的澄清溶液。显而易见, 这种高分散的团簇将可 以提供更多的催化活性位点, 正如他们的测试结 果所证实的, 后者的产氢活性达到前者的十多倍。 他们认为这种截然不同的溶液分散行为, 可能是 来源其不同的簇核结构, 包括锆、钛与邻菲罗啉配 体的不同结合能力。

该研究工作已在物理化学学报上在线发 表(doi: 10.3866/PKU.WHXB201711131), 为特刊
“原子水平上精确控制纳米簇和纳米粒子” 邀请 的原创文章 ${ }^{8}$ 。该工作成功将深共熔溶剂热合成引 入到锆及钛锆混合氧簇的合成, 报道了由苯酚和 邻菲罗啉等共轭配体保护的 $\mathrm{Zr}_{6}$ 以及 $\mathrm{Ti}_{11} \mathrm{Zr}_{4}$ 团簇 的结构与物理性能, 对丰富此类团簇化合物的合 成策略及深入理解其结构-催化活性之间的构效 关系具有重要意义。

\section{References}

(1) Coppens, P.; Chen, Y.; Trzop, E. Chem. Rev. 2014, 114, 9645. doi: $10.1021 / \mathrm{cr} 400724 \mathrm{e}$

(2) Lv, Y.; Cheng, J.; Steiner, A.; Gan, L.; Wright, D. S. Angew. Chem. Int. Ed. 2014, 53, 1934. doi: 10.1002/anie.201307721

(3) Li, N.; Matthews, P. D.; Luob, H. K.; Wright, D. S. Chem. Commun. 2016, 52, 11180. doi: 10.1039/C6CC03788G

(4) Rozes, L.; Sanchez, C. Chem. Soc. Rev. 2011, 40, 1006 doi: 10.1039/C0CS00137F

(5) Fang, W. H.; Zhang, L.; Zhang, J. Chem. Soc. Rev. 2018, 47, 404. doi: $10.1039 / \mathrm{C} 7 \mathrm{CS} 00511 \mathrm{C}$

(6) Zhang, G.; Liu, C.; Long, D. L.; Cronin, L.; Tung, C. H.; Wang, Y. J. Am. Chem. Soc. 2016, 138, 11097. doi: 10.1021/ jacs.6b06290

(7) Narayanam, N.; Fang, W. H.; Chintakrinda, K.; Zhang, L.; Zhang, J. Chem. Commun. 2017, 53, 8078. doi: 10.1039/c7cc04388k

(8) Narayanam, N.; Chintakrinda, K.; Fang, W. H.; Zhang, L.; Zhang, J. Acta Phys. -Chim. Sin. 2018, 34 (7), 781. [Narayanam, N., Chintakrinda, K., 方伟慧, 张否, 张健. 物理化学学报, 2018, 34 (7), 781.] doi: 10.3866/PKU.WHXB201711131 